\title{
Astrocytes and Therapeutics for Parkinson's Disease
}

\author{
Phillip M. Rappold and Kim Tieu \\ Department of Neurology, Center for Translational Neuromedicine, University of Rochester School of Medicine and Dentistry, \\ Rochester, New York 14642
}

\begin{abstract}
Summary: Astrocytes play direct, active, and critical roles in mediating neuronal survival and function in various neurodegenerative disorders. This role of astrocytes is well illustrated in amyotrophic lateral sclerosis (ALS), in which the removal of glutamate from the extracellular space by astrocytes confers neuroprotection, whereas astrocytic release of soluble toxic molecules promotes neurodegeneration. In recent years, this context-dependent dual role of astrocytes has also been documented in experimental models of Parkinson's disease. The present review addresses these studies and some potential mechanisms by which astrocytes may influence the neurodegenerative processes in Parkin-
\end{abstract}

son's disease, and in particular examines how astrocytes confer neuroprotection either through the removal of toxic molecules from the extracellular space or through the release of trophic factors and antioxidant molecules. In contrast, under pathological conditions, astrocytes release proinflammatory cytokines and other toxic molecules that are detrimental to dopaminergic neurons. These emerging roles of astrocytes in the pathogenesis of Parkinson's disease constitute an exciting development with promising novel therapeutic targets. Key Words: Parkinson's disease, astrocytes, neurodegeneration, dopamine, glial-neuronal interactions, neurodegenerative diseases.

\section{INTRODUCTION}

Parkinson's disease (PD), the second most common chronic neurodegenerative disorder, is characterized primarily by the death of dopaminergic neurons in the substantia nigra pars compacta ( $\mathrm{SNpc}$ ) and the presence of intracellular protein aggregates known as Lewy bodies. ${ }^{1,2}$ When the concentration of striatal dopamine is reduced below a threshold level of $70-80 \%$, as a result of the death of the nigral dopaminergic neurons, symptoms of PD emerge. ${ }^{1}$ The predominant motor abnormalities seen in patients with PD include bradykinesia, resting tremor, rigidity, and postural instability.

Both environmental and genetic factors play a critical role in the etiology of PD. The role for environmental toxicants has been hypothesized based on observations that parkinsonism can be caused by postencephalitic infection, ${ }^{3,4}$ by accidental injection of 1-methyl-4-phenyl1,2,3,6-tetrahydropyridine (MPTP), ${ }^{5}$ and by chronic manganese intoxication. ${ }^{6}$ Consistent with this view, human epidemiological studies have shown that rural living

Address correspondence and reprint requests to: Kim Tieu, Ph.D., Department of Neurology in the Center for Translational Neuromedicine, University of Rochester School of Medicine, 575 Elmwood Avenue, Box 645, Rochester, NY, 14642. E-mail: kim_tieu@urmc. rochester.edu. and exposure to herbicides, pesticides, and heavy metals increase the risk of PD. ${ }^{7-11}$ In the last few years, exciting discoveries have been made in genetic mutations linked to early-onset and sporadic PD. ${ }^{12,13}$ Studies of these autosomal dominant genes (the $\alpha$-synuclein gene, SNCA; $L R R K 2$, previously $P A R K 8$ ) and autosomal recessive genes (PARK2, alias parkin; PARK7, alias DJ1; PINK1, previously PARK6; ATP13A2, previously PARK9) have provided significant insights into mechanisms of cell death in nigral dopaminergic neurons, such as mitochondrial dysfunction, oxidative stress, neuroinflammation, and insufficient autophagic or proteasomal protein degradation.

To study the mechanisms of neurodegeneration in PD, researchers have focused their attention primarily on the affected nigral dopaminergic neurons. The neighboring glial cells, however, play a significant role in the demise of these neurons. For example, by releasing neuroinflammatory factors, activated microglia are detrimental to nigral dopaminergic neurons. This topic has been extensively reviewed elsewhere. ${ }^{14,15}$ In this review article, we will focus our attention on recent studies highlighting astrocytes as emerging potential non-cell autonomous modulators of dopaminergic neurodegeneration. Where relevant, potential neurotherapeutic strategies targeting astrocytes will also be explored. 


\section{ASTROCYTES AND NEURODEGENERATION}

Astrocytes, the most numerous of glial cells, constitute a major class of cells in the mammalian brain and outnumber neurons by severalfold in the human brain. ${ }^{16}$ Not only are astrocytes present in all regions of the brain, but they also appear to be organized in strategic positions that are in close contact with neuronal structures. The past stereotype of astrocytes as a kind of glue that provides mere structural support to hold the neurons in place is being increasingly revised with the recognition that these glial cells actively play critical and integral roles in mediating the physiologic and pathologic states of neurons. ${ }^{17,18}$ The neuroprotective and neurodegenerative roles of astrocytes depend largely on the molecules that they release into and take up from the extracellular space, which is the microenvironment that astrocytes and neurons share. For example, astrocytes can release and supply neurons with neurotrophic factors such as nerve growth factor (NGF), neurotrophin-3, and basic fibroblast growth factor (bFGF), as well as metabolic substrates such as lactate and the antioxidant glutathione for the survival and proper functioning of neurons. Astrocytes also confer neuroprotection by siphoning away excess extracellular excitotoxic agents, such as glutamate, potassium, and calcium. On the other hand, when astrocytes undergo a state of gliosis in response to neuronal injury or toxic insults, together with microglia they release cytokines and chemokines that are deleterious to neurons. In the following sections, we will examine more closely recent studies on the dual role of astrocytes in neuronal survival and function in the context of PD.

\section{ASTROCYTES CONFER NEUROPROTECTION BY RELEASING SOLUBLE MOLECULES}

\section{Glutathione}

The brain is particularly vulnerable to oxidative stress, largely because of its high metabolic rate. Although through the course of evolution mitochondria have provided eukaryotic cells with a very efficient method of aerobic metabolism, an estimated $1-2 \%$ of this consumed oxygen is converted to reactive oxygen species (ROS), rather than to water. ${ }^{19}$ This inability of the mitochondrial respiratory chain to completely reduce oxygen to water, coupled with a high rate of oxygen consumption, contributes to high ROS production by mitochondria. Under a pathological state of mitochondrial dysfunction, this rate may increase even more dramatically, as a result of leakage of electrons from the electron transport chain and the subsequent reduction of oxygen to superoxide. Consistent with this observation, oxidative stress has been proposed as a pathogenic mechanism in neurodegenerative disorders such as PD, in which mitochondrial defects have been reported. ${ }^{20,21}$ Intrinsi- cally, the brain is equipped with defense mechanisms against reactive oxygen species (ROS), such as the antioxidant enzymes superoxide dismutase, glutathione peroxidase, and catalase, as well as the antioxidant glutathione (GSH). ${ }^{22}$

Glutathione is synthesized intracellularly by $\gamma$-glutamylcysteine synthetase and GSH synthetase. The concentration of GSH in astrocytes $(\sim 3.8 \mathrm{mmol} / \mathrm{L})$ is estimated to be higher than that of neurons $(\sim 2.5 \mathrm{mmol} /$ L), ${ }^{23,24}$ probably as a result of higher specific activity of the $\gamma$-glutamylcysteine synthetase in astrocytes. ${ }^{25} \mathrm{Be}$ cause of their close proximity, however, astrocytes are able to share their GSH with the neighboring neurons by releasing this antioxidant through the transporter multidrug resistance protein 1 (MRP1) into extracellular space. $^{26}$ From this shared microenvironment, GSH is cleaved by $\gamma$-glutamyltranspeptidase on astrocytic plasma membrane to generate precursors for neuronal GSH synthesis. This release appears to be a uniquely astrocytic modality; it has been demonstrated that neurons, microglia, and oligodendrocytes do not readily release $\mathrm{GSH},{ }^{26}$ probably due to low levels of MRP1 in these cell types. ${ }^{27}$ Thus, astrocytes are the major contributor to the extracellular levels of GSH. Overall, both intracellular and extracellular levels of GSH influence neuronal survival. Consistent with the neuroprotective role of GSH, reductions in GSH levels have been reported in patients and animal models of various neurodegenerative disorders. The best available data implicating deficient GSH as a pathogenic factor are for PD. ${ }^{28}$

The GSH content in the substantia nigra of PD patients has been observed to be significantly reduced $(\sim 40 \%){ }^{29}$ At the cellular level, surviving nigral dopaminergic neurons display a significant loss of GSH. ${ }^{30}$ Although the mechanism of this reduction has not been established, it is unlikely to be secondary to neuronal loss or drug treatment. $^{28}$ In genetic models of PD, astrocytes aged in cultures from parkin-knockout mice have lower levels of GSH than those from wild-type animals. ${ }^{31}$ It is not clear, however, whether depletion in GSH alone may be sufficient to induce cell death, because buthionine sulfoximine, an inhibitor of $\gamma$-glutamylcysteine synthetase, does not kill dopaminergic cells. ${ }^{32}$ It is possible that GSH depletion enhances cell death under pathological conditions. ${ }^{33}$ Consistent with this argument, GSH depletion or its loss of function enhances dopaminergic cell death in Drosophila models with parkin mutation ${ }^{34}$ or overexpression of $\alpha$-synuclein, ${ }^{35}$ two genetic mutations in PD.

Recognizing the critical role played by GSH in neuronal survival, attempts have been made to restore GSH levels in PD therapy. However, a recent randomized, double-blind clinical trial of parenteral GSH administration in patients with PD failed to show any clinical benefits. ${ }^{36}$ Because the blood-brain barrier permeability to GSH is low, ${ }^{37-39}$ this approach may not be ideal for 
delivering GSH to dopaminergic neurons. An alternative strategy would be to target the molecules regulating the brain GSH system to maintain or even enhance the antioxidant capabilities of dopaminergic neurons. One such example that has gained attention recently is the NF-E2related factor (Nrf2) transcription factor, which is known to regulate the expression of several cytoprotective genes containing the cis-acting enhancer sequence referred to as the antioxidant response element (ARE).$^{40}$ Glutathione S-transferase, which conjugates GSH to electrophilic compounds, is one of such ARE-regulated genes in astrocytes. ${ }^{41}$ Under physiologic conditions, Nrf2 transcriptional activity is kept to a minimum by the cytosolic binding Kelch-like ECH-associated protein 1 (Keap1), which targets Nrf2 for ubiquitination and subsequent proteasomal degradation. ${ }^{42}$ Under cellular stress, Nrf2 is stabilized and translocated to the nucleus, where it dimerizes with other transcriptional molecules and promotes the transcription of ARE-containing genes involved in glutathione, iron, and NADPH homeostasis in astrocytes. ${ }^{43}$ In several in vitro and in vivo models, Nrf2 has been found to be preferentially induced in astrocytes. ${ }^{43-45}$ Furthermore, Chen et al. ${ }^{46}$ have demonstrated in vivo that astrocyte-confined $\mathrm{Nrf} 2$ overexpression is sufficient to provide neuroprotection, whereas loss of Nrf2 function enhances neuronal degeneration in a murine MPTP model of PD.

The connection of this Nrf2 system to the pathophysiology of PD has been further strengthened by the discovery that the PARK7 gene (encoding the DJ-1 protein) is necessary for the transcriptional activity of Nrf2. ${ }^{47}$ Homozygous mutations in PARK 7 are known to cause a recessive form of early-onset familial PD. ${ }^{48,49}$ Although the mechanisms are incompletely understood, expression of DJ-1 confers striking protection against cellular insults. DJ-1 is expressed primarily in astrocytes in the normal human CNS and is strongly upregulated in PD. ${ }^{50}$ Primary astroglial cultures isolated from $\mathrm{Park}^{-1-}$ mice strongly suggest that, besides promoting the expression of antioxidant genes, DJ-1 also plays an important role in the suppression of proinflammatory responses. ${ }^{51}$ Hence, restoring or enhancing the function of the astrocytic DJ1-Nrf2 pathway may represents a therapeutic strategy for PD patients.

\section{Trophic factors}

Astrocytes produce a variety of trophic factors that can support neuronal function. For example, basic fibroblast growth factor (bFGF or FGF-2), ${ }^{52}$ glial cell line-derived neurotrophic factor (GDNF), ${ }^{53,54}$ and mesencephalic astrocyte-derived neurotrophic factor (MANF) ${ }^{55}$ have been shown to be protective in PD animal models. Among these trophic factors, GDNF has been most extensively studied and has been found to confer the most protection of dopaminergic neurons. ${ }^{56}$ However, the success of translation of these findings to the clinic has been controversial. The side effects and conflicting results regarding effectiveness have somewhat dampened enthusiasm for these molecules. ${ }^{57,58}$ Because these molecules are rapidly degraded and cannot permeate the blood-brain barrier, inadequate drug delivery to appropriate target neurons may contribute to these negative results. Indeed, when Salvatore et al. ${ }^{59}$ used the same intraputamenal infusion protocol as had been used in a failed human clinical trial of GDNF, they found that GDNF levels were highest in the area surrounding the catheter and were exponentially reduced with increasing distance from the source of release, resulting in less than $10 \%$ of the putamen in the human subjects being exposed to GDNF. In the murine 6-hydroxydopamine (6-OHDA) model,${ }^{60}$ intrastriatal injection of MANF was found to be neuroprotective and have superior tissue distribution compared to GDNF. MANF, therefore, could be a significant therapeutic treatment for PD.

\section{ASTROCYTES CONFER NEUROPROTECTION BY REMOVING TOXIC MOLECULES}

\section{Clearance of extracellular $\alpha$-synuclein}

$\alpha$-Synuclein is a small, $14-\mathrm{kDa}$ protein that is abundantly available in presynaptic terminals. Consistent with its cellular location, this protein has been shown to be involved in modulating synaptic vesicle function. ${ }^{61} \mathrm{Al}-$ though the exact physiological function of $\alpha$-synuclein is incompletely understood, missense mutations or gene multiplication mutations leading to higher levels of wildtype synuclein cause autosomal-dominant PD. ${ }^{62-65}$ $\alpha$-Synuclein is natively unfolded in solution ${ }^{66}$; however, it has a propensity to form aggregates under various pathological conditions. ${ }^{67}$ The aggregated and insoluble fibrillar form of $\alpha$-synuclein constitutes a major component of the intracellular proteinaceous inclusions called Lewy bodies. Although it is still a topic of debate whether these insoluble protein aggregates are neurotoxic, neuroprotective, or incidental, the soluble oligomeric species are likely neurotoxic.

$\alpha$-Synuclein has been a protein of great interest since it was discovered in the 1990s as the first genetic mutation in PD. ${ }^{62-65}$ It has recently gained additional attention subsequent to the first post-mortem analyses in patients with PD receiving transplanted embryonic dopaminergic neurons. In three of four patients who died 11 to 16 years after transplantation, $\alpha$-synuclein positive protein aggregates were present in grafted neurons; however, these aggregates were not detectable in four patients who died 4 to 9 years after the transplantation. ${ }^{68-70}$ One possible explanation for these observations is that $\alpha$-synuclein can spread from the dying host neurons to the grafted ones in a time-dependent manner. ${ }^{71}$ Shortly after the reports of these human studies, Lee and col- 
leagues demonstrated in cell culture and animal models that $\alpha$-synuclein indeed is transmittable from neuron to neuron ${ }^{72}$ and from neuron to astrocytes. ${ }^{73}$ This unusual property has led investigators to propose that synuclein possesses "prion-like" properties. ${ }^{74,75}$ The adverse effects of $\alpha$-synuclein in neurons have been extensively studied over the years, with increased levels inversely correlating with tyrosine hydroxylase immunoreactivity in nigral dopaminergic neurons. ${ }^{76}$ In astrocytes, however, the effects of $\alpha$-synuclein remain largely unexplored.

To demonstrate the transfer of $\alpha$-synuclein from neuron to astrocytes, Lee and colleagues either treated primary astrocytes with medium containing secreted $\alpha$-synuclein from human neuroblastoma SH-SY5Y cells stably overexpressing this protein or co-cultured astrocytes with these stable cells. ${ }^{73}$ In addition to detecting $\alpha$-synuclein transferred to astrocytes under both conditions, the formation of large astrocytic Lewy bodies was observed in a time dependent manner. In vivo studies using transgenic mice overexpressing $\alpha$-synuclein driven by the neuronal promoter PDGF $\beta$ yielded additional supporting evidence. In these animals, $\alpha$-synuclein immunoreactivity was detected in both neurons and astrocytes. Given that astrocytes do not express $\alpha$-synuclein in these animals, the detection of $\alpha$-synuclein in these cells suggests it was derived from neurons and subsequently transferred intercellularly. ${ }^{73}$

Once inside astrocytes, $\alpha$-synuclein is degraded through the lysosomal pathway. ${ }^{73}$ Thus, astrocytes may confer neuroprotection to dopaminergic neurons by clearing excess extracellular toxic $\alpha$-synuclein. A similar protective role of astrocytes has been demonstrated in models of Alzheimer's disease, where they have been shown to uptake and degrade extracellular $\beta$-amyloid protein. ${ }^{77,78}$ Thus, strategies aimed at enhancing the removal and degradation of $\alpha$-synuclein by astrocytes may be therapeutic. However, research to identify mechanisms of $\alpha$-synuclein transport is still in its infancy.

\section{ASTROCYTES INDUCE NEURODEGENERATION BY RELEASING TOXIC MOLECULES}

\section{Toxic molecules mediated by monoamine oxidase-B metabolism}

The first and most direct evidence to date for the involvement of astrocytes in the loss of nigrostriatal dopaminergic is the discovery of 1-methyl-4-phenyl1,2,3,6-tetrahydropyridine (MPTP) induced parkinsonism. ${ }^{5}$ In 1982, a group of drug addicts in California was rushed to the emergency room presenting with a severe bradykinetic and rigid syndrome, ${ }^{5}$ similar to that seen in PD patients. Subsequent investigations led to the discovery that, a few days earlier, these patients had self-ad- ministered MPTP-contaminated synthetic meperidine. ${ }^{79}$ The movement abnormalities exhibited in these patients were L-DOPA responsive, as are those seen in PD patients. Since the discovery of parkinsonism caused by MPTP in these patients, this neurotoxicant has been used extensively as a model of PD in nonhuman primates, as well as in other mammalian species. ${ }^{79,80}$ Recently, one of the surviving patients from the original 1982 cases showed a significant clinical improvement when treated with deep brain stimulation, suggesting that the MPTP model may be valid for the study of neurodegeneration in PD. ${ }^{81}$

The mechanism of MPTP toxicity has been studied extensively and is quite well characterized. ${ }^{1}$ MPTP is a lipophilic prototoxin that rapidly crosses the blood-brain barrier. In astrocytes, MPTP is metabolized by monoamine oxidase-B (MAO-B) and subsequently converted to the active toxic cation of 1-methyl-4-phenylpyridinium $\left(\mathrm{MPP}^{+}\right)$. We recently demonstrated that, from astrocytes, $\mathrm{MPP}^{+}$is extruded into the extracellular space through the organic cation transporter $3 .^{82}$ Because $\mathrm{MPP}^{+}$has a high affinity for the dopamine transporter (DAT), it is taken up by the neighboring dopaminergic neurons and terminals, where it induces neurotoxicity via its inhibition of complex I of the mitochondrial electron transport chain. In addition to its affinity for DAT, MPP ${ }^{+}$ also has high affinity for noradrenergic and serotonergic uptake transporters ${ }^{83,84}$; however, according to most studies, monoaminergic systems other than the nigrostriatal pathway are not prime targets for MPTP neurotoxicity. Nonetheless, direct injection of $\mathrm{MPP}^{+}$does produce damage to these monoaminergic nuclei. ${ }^{85}$ Taken together, these studies suggest that perhaps the release of $\mathrm{MPP}^{+}$is not evenly distributed in the brain regions that are and are not affected by MPTP toxicity. Consistent with this theory, we discovered that the expression of organic cation transporter (Oct3) is selective to the group of astrocytes in the nigrostriatal regions but not in those residing in the brain regions that are not sensitive to MPTP toxicity. In vivo microdialysis results show that inactivation of this transporter attenuates the release of $\mathrm{MPP}^{+}$into extracellular space, consistent with the observation of less nigral neurodegeneration in MPTP injected $O c t 3^{-1-}$ mice. ${ }^{82}$ Thus, the selective expression of Oct3 in nigrostriatal astrocytes contributes to the selective neurodegeneration of dopaminergic neurons in this region.

Although the metabolism of MPTP to $\mathrm{MPP}^{+}$by MAO-B is no longer a risk for PD, it is possible that other endogenous toxic molecules maybe generated through this mechanism. For instance, the degradation of dopamine by MAO-B generates reactive dopamine quinones and other oxidative species. Tetra-isoquinoline has also been demonstrated to be metabolized by this enzyme in astrocytes to form isoquinolinium cations ${ }^{86}$ 
These lipophilic compounds, which have been linked to parkinsonism, are present in the environment, in foodstuffs, and in the brain through endogenous formation.

Primarily based on observations that MAO-B plays a critical role in MPTP toxicity and generates reactive oxidative species through dopamine metabolism, this enzyme has been a target for the treatment of PD. With aging, which is a consistent risk factor for PD, the expression levels of MAO-B also increase and its activity correlates positively with the degree of cell loss in the substantia nigra. ${ }^{87-89}$ More direct evidence has been shown in transgenic mice with inducible overexpression of MAO-B in astrocytes under the GFAP promoter. ${ }^{90}$ These mice were found to have age-related motor dysfunction that paralleled degeneration of neurons in the SNpc, suggesting that increased MAO-B expression and activity is sufficient to induce a parkinsonian phenotype. Conversely, smokers, who consistently have been reported to have a lower risk of developing $\mathrm{PD}$, have significantly decreased MAO-B activity. ${ }^{91,92}$ Based on such lines of evidence, the MAO-B inhibitors selegiline and rasagiline have been developed and routinely used as monotherapy or as an adjunct to L-DOPA in the treatment of PD. The efficacy of selegiline has been extensively assessed in multicenter studies, including the DATATOP trial. ${ }^{93-95}$ MAO-B inhibitors have been found to be beneficial for ameliorating motor symptoms in PD and perhaps, although this is still controversial, offer neuroprotection as well through both the inhibition of MAO-B and independent and incompletely understood antiapoptotic effects of the propargylamine moiety common to these molecules. ${ }^{96}$

\section{Proinflammatory cytokines}

A review of the literature quickly reveals that the majority of findings from research on neuroinflammation points toward microglia. In fact, most, if not all, articles discussing neuroinflammation focus only on microglia. However, several lines of evidence suggest that astrocytes do release proinflammatory cytokines under pathological conditions. For instance, as already noted, a recent study shows that astrocytes are capable of removing extracellular $\alpha$-synuclein released from neurons. ${ }^{73} \mathrm{Al}$ though this uptake and subsequent degradation of $\alpha$-synuclein in astrocytes may confer initial protection to neurons, once the accumulation of $\alpha$-synuclein exceeds the degradation capacity of astrocytes, then aggregates of $\alpha$-synuclein start to form in astrocytes. This scenario has been demonstrated when bafilomycin A1, a lysosomal inhibitor, increases the formation of detergent-insoluble $\alpha$-synuclein in astrocytes. ${ }^{73}$ Under this pathological condition, upregulation of transcripts of inflammatory cytokines such as IL- $1 \alpha$, IL- $1 \beta$, and IL- 6 and the release of TNF- $\alpha$ and IL- 6 is detected. ${ }^{73}$ Consistent with this toxic role of $\alpha$-synuclein, the presence of $\alpha$-synuclein in astrocytes correlates with nigral neuronal cell death. ${ }^{97}$

In addition to direct release of proinflammatory cytokines, astrocytes can also be activated by cytokines such as TNF- $\alpha$ and IL- $1 \beta$ from microglia, leading to production of reactive oxygen and nitrogen species. ${ }^{98}$ For example, a recent study demonstrated in a coculture model that astrocytes enhance microglial inflammatory responses through an NF- $\kappa \mathrm{B}$ dependent mechanism, leading to more dopaminergic toxicity. ${ }^{98}$ The authors further showed that these effects are mediated by a novel function of Nurr1, a transcription factor crucial for the development and maintenance of dopaminergic neurons in the mesencephalon. ${ }^{99-101}$ Notably, mutations in the NR4A2 gene (previously NURRI) are associated with rare familial PD. ${ }^{102-104}$ These mutations lead to a reduction in NR4A2 mRNA levels in affected PD patients and cells transfected with mutant NR4A2. ${ }^{102}$ Thus activating the orphan nuclear receptor NURR1 protein produces both anti-inflammatory effects and improvement in dopaminergic development and function. The development of small molecule agonists for the NURR1 protein to probe this therapeutic target are underway. ${ }^{105}$

\section{Purinergic molecules}

Purinergic signaling, subject of a rapidly growing field of study, is now recognized as a prevalent and complex form of intercellular communication between glia and neurons. ${ }^{106}$ Astrocytes serve as an important source of extracellular purines. Adenosine triphosphate is released by astrocytes through mechanisms such as exocytosis, connexin or pannexin hemichannels, volume-regulated chloride channels, and $\mathrm{P} 2 \mathrm{X}_{7}$ receptors. ${ }^{106}$ After release, ATP is rapidly degraded by a series of ectonucleotidases to ADP, AMP, and adenosine. In addition to this indirect mechanism, adenosine can also be released directly by astrocytes into extracellular space via equilibrative nucleoside transporters. ${ }^{107}$ Purinergic receptors are broadly divided into two families, with one preferentially stimulated by adenosine (P1 or A receptors) and the other preferentially stimulated by ATP (P2 receptors). All adenosine receptors are G-protein coupled, whereas the $\mathrm{P} 2$ family is further subdivided into ionotropic $(\mathrm{P} 2 \mathrm{X})$ and metabotropic (P2Y) classes. In contrast to $\mathrm{A}_{1}, \mathrm{~A}_{2 \mathrm{~B}}$, and $\mathrm{A}_{3 \mathrm{~A}}$ adenosine receptors, which have a relatively wide pattern of distribution in the CNS, the $\mathrm{A}_{2 \mathrm{~A}}$ receptors are more restricted to the basal ganglia and are best characterized for $\mathrm{PD}^{108-110}$; however, $\mathrm{P} 2$ receptors may also be involved in neurodegeneration. ${ }^{111}$

GABAergic medium spiny neurons constitute the majority of neurons in the striatum, the primary input nucleus of the basal ganglia receiving dopaminergic afferents from the $\mathrm{SNpc}$ and glutamatergic afferents from virtually all areas of cortex. There are two anatomically and functionally distinct types of medium spiny neurons, 
although they are equivalent in numbers and uniformly distributed in the striatum. Striatonigral medium spiny neurons (which represent the direct pathway) express substance $\mathrm{P}$ and $\mathrm{D}_{1}$ dopamine receptors. These neurons project to the internal segment of the globus pallidus (GPi) and the substantia nigra pars reticulata ( $\mathrm{SNpr}$ ). The GPi and SNpr constitute the primary output nuclei of the basal ganglia and send inhibitory GABAergic projections to the motor nuclei of the thalamus, which in turn is excitatory to motor cortex. The net effect of this direct pathway is to facilitate motor movements by disinhibiting thalamocortical projections to motor cortex. On the other hand, the striatopallidal medium spiny neurons (which represent the indirect pathway) express enkepha- lin, $\mathrm{D}_{2}$ dopamine, and $\mathrm{A}_{2 \mathrm{~A}}$ receptors. They project to the external segment of the globus pallidus (GPe), inhibiting the subthalamic nucleus (STN), which sends excitatory glutamatergic projections to the GPi/SNpr. The net effect of this pathway is to suppress motor movements by inhibiting thalamocortical neurons. The dopaminergic input from the SNpc suppresses the indirect pathway via $D_{2}$ receptors, but stimulates the direct pathway via $D_{1}$ receptors, thus facilitating movement. Hence, any process resulting in striatal dopamine depletion results in a parkinsonian syndrome of bradykinesia, postural instability, and rigidity.

Because $A_{2 A}$ and $D_{2}$ receptors are positively and negatively coupled to adenylate cyclase, respectively,

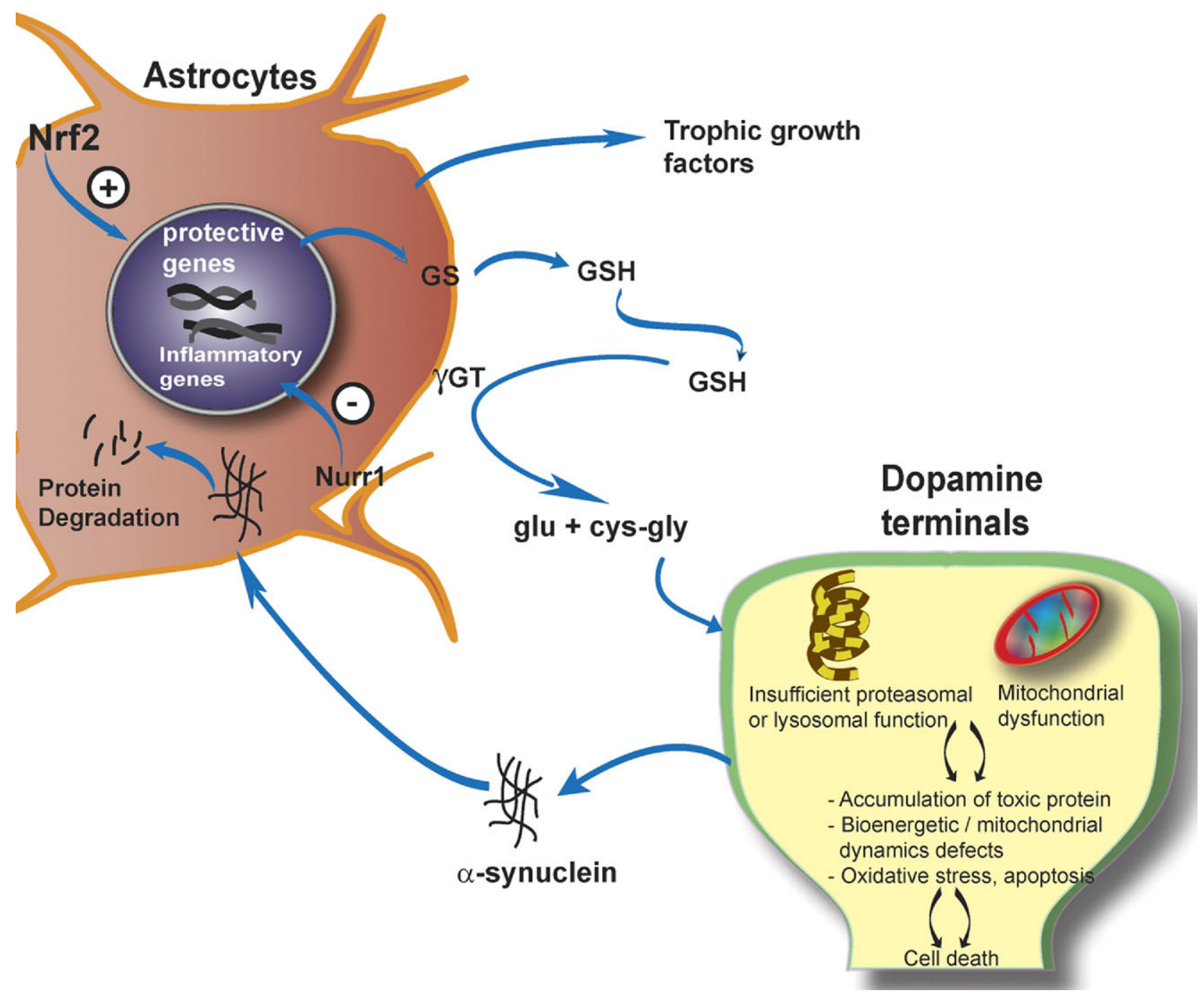

FIG. 1. Potential neuroprotective pathways of astrocytes. Genetic mutations, environmental toxicants, or a combination of both may induce nigral dopaminergic neurotoxicity through mechanisms such as mitochondrial dysfunction and insufficient degradation of misfolded proteins. Astrocytes may mediate neuroprotection through the following pathways. 1) Release of trophic growth factors, such as bFGF, GDNF, and MANF). 2) Release of glutathione (GSH), which is then cleaved by $\gamma$-glutamyltranspeptidase on astrocytic plasma membrane to generate glutamate and cysteinylglycine, which serves as precursors for neuronal GSH synthesis. 3) Activation of the transcription factor Nrf2 leads to expression of genes containing the antioxidant response element (ARE), including $\gamma$-glutamylcysteine synthetase (GS), which is involved in GSH synthesis. 4) Activation of the transcription factor Nurr1, which suppresses the production of inflammatory cytokines. 5) Removal and degradation of cytotoxic molecules, such as $\alpha$-synuclein. 
adenosine and dopamine exert antagonistic effects in modulating the activity of striatopallidal neurons. ${ }^{110}$ The loss of striatal dopamine in PD leads to unopposed adenosine stimulation of this system, resulting in hyperactivity of the indirect pathway and subsequent motor dysfunction characteristic of PD. Blocking the effects of adenosine at $A_{2 A}$ therefore represents an attractive therapeutic target. Consistent with this mechanism, $\mathrm{A}_{2 \mathrm{~A}}$ antagonists enhance $D_{2}$-mediated inhibitory effects on the striatopallidal neurons and increase $\mathrm{D}_{1}$-mediated stimulatory effects on the striatonigral neurons, resulting in alleviation of parkinsonian motor symptoms in rodents ${ }^{112}$ and MPTP-treated nonhuman primates, ${ }^{113}$ as well as in humans. ${ }^{114-116}$
Together these studies strongly support the role of $\mathrm{A}_{2 \mathrm{~A}}$ antagonists as novel nondopaminergic therapy in relieving parkinsonian motor deficits. The beneficial effects of blocking $\mathrm{A}_{2 \mathrm{~A}}$ may even extend to protecting dopaminergic neurons from degeneration. For example, epidemiological studies consistently report that intake of caffeine (a nonselective adenosine receptor antagonist), whether from coffee or noncoffee sources, reduces risk of developing PD. ${ }^{117,118}$ More direct evidence is provided in a mouse model of $\mathrm{PD}$, in which caffeine or genetic deletion of $\mathrm{A}_{2 \mathrm{~A}}$ attenuated MPTP- and 6-OHDA-induced dopaminergic neurotoxicity. ${ }^{19-121}$ The mechanism of this neuroprotection has been attributed to the attenuation of neuroinflammation induced by astrocytes and

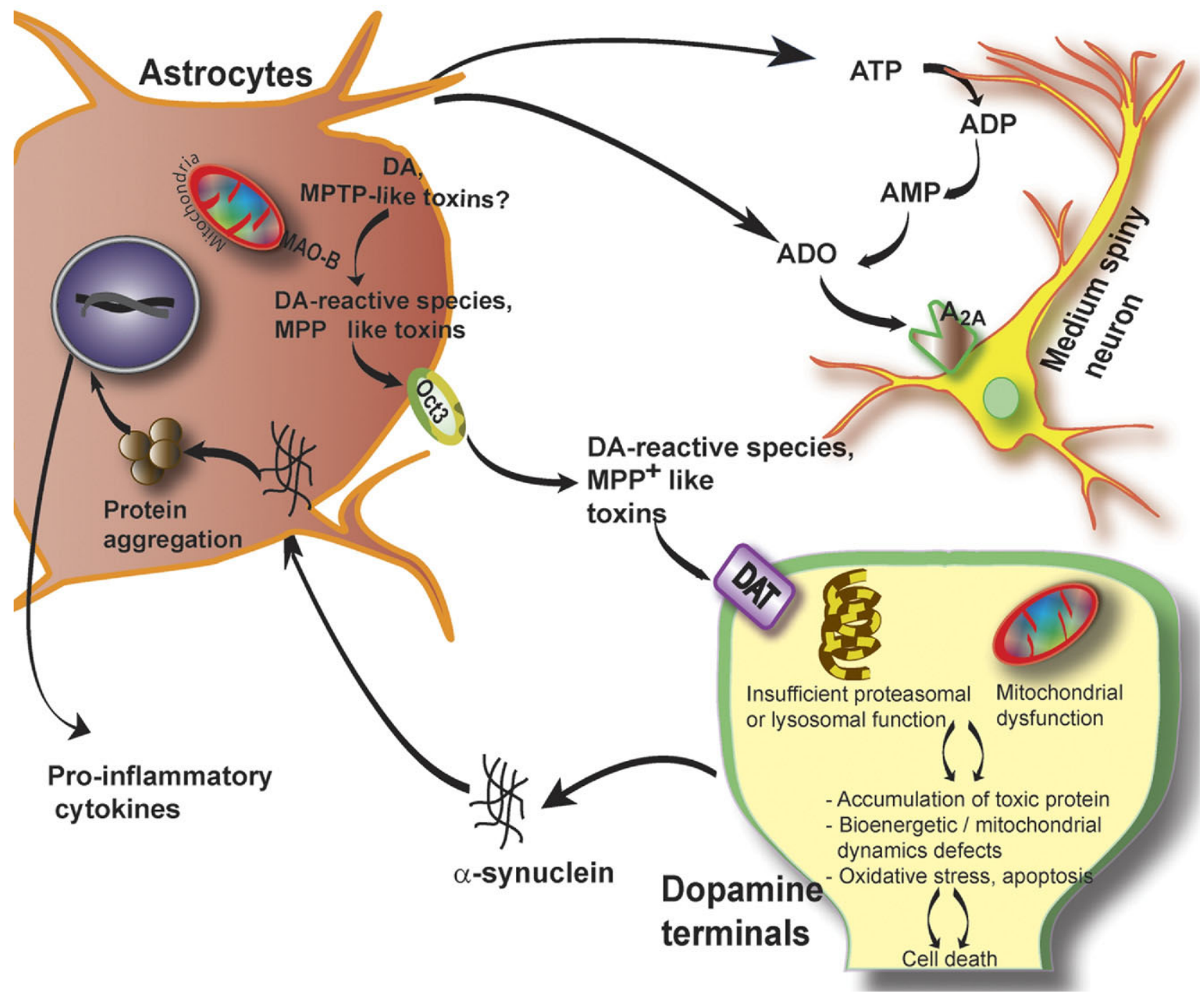

FIG. 2. Potential neurotoxic pathways of astrocytes. Astrocytes may also adversely affect the survival and function of dopaminergic neurons through the following mechanisms: 1) Release of proinflammatory cytokines under pathological conditions such as accumulation of aggregated $\alpha$-synuclein. 2) Monoamine oxidase-B (MAO-B) mediated release of cytotoxic molecules such as dopamine-related oxidants and $\mathrm{MPP}^{+}$-like organic cations through the organic cation transporter (Oct3) into the extracellular space where they are subsequently transported into DA neurons through the dopamine transporter (DAT). 3) Astrocytes can also release adenosine (ADO) directly or indirectly via ATP. ADO may increase movement disorders in patients with PD through the $A_{2 A}$ receptors in striatal medium spiny neurons. 
microglia, as well as the reduced glutamate release from the presynaptic glutamatergic terminals or astrocytes in the striatum. ${ }^{114-116}$ However, additional studies are needed to elucidate the neuroprotective mechanism of $\mathrm{A}_{2 \mathrm{~A}}$ inhibition.

Another relatively unexplored aspect of this system is the cellular and molecular source of extracellular adenosine. Astrocytes remain a likely candidate as the cellular source, by virtue of their well-studied mechanisms of purine release in other brain regions and in disease models. ${ }^{122,123}$ Regardless of the cellular source, the mechanism of release (i.e., exocytotic or transmembrane protein channels) and whether it is adenosine or another precursor molecule (ATP, cAMP) that is released into the extracellular compartment, further investigation is warranted, because they constitute additional potential therapeutic targets.

\section{CONCLUSION}

Since the recognition of PD more than 200 years ago, the primary focus of research in elucidating the mechanisms of cell death in this neurological disorder has been the affected dopaminergic neurons. However, as reviewed in this article and summarized in FIGS. 1 and 2, through diverse pathways of release or removal of protective and neurotoxic molecules, a picture starts to emerge that astrocytes, either directly or indirectly, play a role in survival and function of dopaminergic neurons in experimental models of PD.

One challenge in the study of how astrocytes modulate neurotoxicity in PD is the lack of suitable models of neurodegeneration in this disorder. Despite their popularity, neurotoxins such as MPTP and 6-OHDA still remain acute toxic models, which, understandably, may not accurately model PD pathogenesis. With recent discoveries of genetic mutations in PD, various animal models have been developed; however, these genetic models are not suitable for the study of neurodegeneration. ${ }^{124}$ Animal models expressing these genetic mutations in astrocytes are currently lacking, but if developed might also be useful tools to further investigate the role of astrocytes in pathogenesis and therapeutic development for PD. Despite these obstacles, current evidence supports the idea that astrocytes are fully capable of modulating the survival and function of dopaminergic neurons. Therapeutic strategies targeting astrocytes for PD treatment therefore warrant consideration.

Acknowledgments: This work was supported in part by U.S. National Institutes of Health grants ES014899 and ES17470 (to K.T.) and training grant TL1RR024135 (to P.M.R.). P.M.R. is a trainee in the Medical Scientist Training Program funded by NIH T32 GM07356. The content is solely the responsibility of the authors and does not necessarily represent the official views of the National Institute of General Medical Sciences or NIH.

\section{REFERENCES}

1. Dauer W, Przedborski S. Parkinson's disease: mechanisms and models. Neuron 2003;39:889-909.

2. Lees AJ, Hardy J, Revesz T. Parkinson's disease [Erratum in: Lancet 2009;374:684]. Lancet 2009;373:2055-2066.

3. Krusz JC, Koller WC, Ziegler DK. Historical review: abnormal movements associated with epidemic encephalitis lethargica. Mov Disord 1987;2:137-141.

4. Calne DB, Lees AJ. Late progression of post-encephalitic Parkinson's syndrome. Can J Neurol Sci 1988;15:135-138.

5. Langston JW, Ballard P, Irwin I. Chronic parkinsonism in humans due to a product of meperidine-analog synthesis. Science 1983;219:979-980.

6. Olanow CW. Manganese-induced parkinsonism and Parkinson's disease. Ann N Y Acad Sci 2004;1012:209-223.

7. Tanner CM. Epidemiology of Parkinson's disease. Neurol Clin 1992;10:317-329.

8. Liou HH, Tsai MC, Chen CJ, et al. Environmental risk factors and Parkinson's disease: a case-control study in Taiwan. Neurology 1997;48:1583-1588.

9. Hertzman C, Wiens M, Bowering D, Snow B, Calne D. Parkinson's disease: a case-control study of occupational and environmental risk factors. Am J Ind Med 1990;17:349-355.

10. Ritz BR, Manthripragada AD, Costello S, et al. Dopamine transporter genetic variants and pesticides in Parkinson's disease. Environ Health Perspect 2009;117:964-969.

11. Gatto NM, Cockburn M, Bronstein J, Manthripragada AD, Ritz B. Well-water consumption and Parkinson's disease in rural California. Environ Health Perspect 2009;117:1912-1918.

12. Gupta A, Dawson VL, Dawson TM. What causes cell death in Parkinson's disease? Ann Neurol 2008;64 (Suppl 2):S3-S15.

13. Hardy J, Lewis P, Revesz T, Lees A, Paisan-Ruiz C. The genetics of Parkinson's syndromes: a critical review. Curr Opin Genet Dev 2009;19:254-265.

14. Przedborski S. Neuroinflammation and Parkinson's disease. Handb Clin Neurol 2007;83:535-551.

15. Hirsch EC, Hunot S. Neuroinflammation in Parkinson's disease: a target for neuroprotection? Lancet Neurol 2009;8:382-397.

16. Sherwood CC, Stimpson CD, Raghanti MA, et al. Evolution of increased glia-neuron ratios in the human frontal cortex. Proc Natl Acad Sci U S A 2006;103:13606-13611.

17. Mena MA, García de Yébenes J. Glial cells as players in parkinsonism: the "good," the "bad," and the "mysterious" glia. Neuroscientist 2008;14:544-560.

18. Heneka MT, Rodríguez JJ, Verkhratsky A. Neuroglia in neurodegeneration. Brain Res Rev 2010;63:189-211.

19. Richter C. Reactive oxygen and DNA damage in mitochondria. Mutat Res 1992;275:249-255.

20. Beal MF. Oxidatively modified proteins in aging and disease. Free Radic Biol Med 2002;32:797-803.

21. Malkus KA, Tsika E, Ischiropoulos H. Oxidative modifications, mitochondrial dysfunction, and impaired protein degradation in Parkinson's disease: how neurons are lost in the Bermuda triangle. Mol Neurodegener 2009;4:24.

22. Ballatori N, Krance SM, Notenboom S, Shi S, Tieu K, Hammond $\mathrm{CL}$. Glutathione dysregulation and the etiology and progression of human diseases. Biol Chem 2009;390:191-214.

23. Rice ME, Russo-Menna I. Differential compartmentalization of brain ascorbate and glutathione between neurons and glia. Neuroscience 1998;82:1213-1223.

24. Bolanos JP, Heales SJ, Land JM, Clark JB. Effect of peroxynitrite on the mitochondrial respiratory chain: differential susceptibility of neurones and astrocytes in primary culture. J Neurochem 1995; 64:1965-1972.

25. Gegg ME, Beltran B, Salas-Pino S, et al. Differential effect of nitric oxide on glutathione metabolism and mitochondrial function in astrocytes and neurones: implications for neuroprotection/ neurodegeneration? J Neurochem 2003;86:228-237.

26. Hirrlinger J, Schulz JB, Dringen R. Glutathione release from cultured brain cells: multidrug resistance protein 1 mediates the release of GSH from rat astroglial cells. J Neurosci Res 2002;69: 318-326. 
27. Hirrlinger J, König J, Dringen R. Expression of mRNAs of multidrug resistance proteins (Mrps) in cultured rat astrocytes, oligodendrocytes, microglial cells and neurones. J Neurochem 2002; 82:716-719.

28. Zeevalk GD, Razmpour R, Bernard LP. Glutathione and Parkinson's disease: is this the elephant in the room? Biomed Pharmacother 2008;62:236-249.

29. Sian J, Dexter DT, Lees AJ, et al. Alterations in glutathione levels in Parkinson's disease and other neurodegenerative disorders affecting basal ganglia. Ann Neurol 1994;36:348-355.

30. Pearce RK, Owen A, Daniel S, Jenner P, Marsden CD. Alterations in the distribution of glutathione in the substantia nigra in Parkinson's disease. J Neural Transm 1997;104:661-677.

31. Solano RM, Casarejos MJ, Menéndez-Cuervo J, et al. Glial dysfunction in parkin null mice: effects of aging. J Neurosci 2008; 28:598-611.

32. Toffa S, Kunikowska GM, Zeng BY, Jenner P, Marsden CD. Glutathione depletion in rat brain does not cause nigrostriatal pathway degeneration. J Neural Transm 1997;104:67-75.

33. Zeevalk GD, Bernard LP, Sinha C, Ehrhart J, Nicklas WJ. Excitotoxicity and oxidative stress during inhibition of energy metabolism. Dev Neurosci 1998;20:444-453.

34. Whitworth AJ, Theodore DA, Greene JC, Benes H, Wes PD, Pallanck LJ. Increased glutathione S-transferase activity rescues dopaminergic neuron loss in a Drosophila model of Parkinson's disease. Proc Natl Acad Sci U S A 2005;102:8024-8029.

35. Trinh K, Moore K, Wes PD, et al. Induction of the phase II detoxification pathway suppresses neuron loss in Drosophila models of Parkinson's disease. J Neurosci 2008;28:465-472.

36. Hauser RA, Lyons KE, McClain T, Carter S, Perlmutter D. Randomized, double-blind, pilot evaluation of intravenous glutathione in Parkinson's disease. Mov Disord 2009;24:979-983.

37. Cornford EM, Braun LD, Crane PD, Oldendorf WH. Bloodbrain barrier restriction of peptides and the low uptake of enkephalins. Endocrinology 1978;103:1297-1303.

38. Kannan R, Kuhlenkamp JF, Jeandidier E, Trinh H, Ookhtens M, Kaplowitz N. Evidence for carrier-mediated transport of glutathione across the blood-brain barrier in the rat. J Clin Invest 1990; 85:2009-2013.

39. McLellan LI, Lewis AD, Hall DJ, Ansell JD, Wolf CR. Uptake and distribution of $N$-acetylcysteine in mice: tissue-specific effects on glutathione concentrations. Carcinogenesis 1995;16: 2099-2106.

40. Itoh K, Chiba T, Takahashi S, et al. An Nrf2/small Maf heterodimer mediates the induction of phase II detoxifying enzyme genes through antioxidant response elements. Biochem Biophys Res Commun 1997;236:313-322.

41. Vargas MR, Johnson JA. The Nrf2-ARE cytoprotective pathway in astrocytes. Expert Rev Mol Med 2009;11:e17.

42. Nguyen T, Nioi P, Pickett CB. The Nrf2-antioxidant response element signaling pathway and its activation by oxidative stress. J Biol Chem 2009;284:13291-13295.

43. Lee JM, Calkins MJ, Chan K, Kan YW, Johnson JA. Identification of the NF-E2-related factor-2-dependent genes conferring protection against oxidative stress in primary cortical astrocytes using oligonucleotide microarray analysis. J Biol Chem 2003; 278:12029-12038.

44. Kraft AD, Johnson DA, Johnson JA. Nuclear factor E2-related factor 2-dependent antioxidant response element activation by tert-butylhydroquinone and sulforaphane occurring preferentially in astrocytes conditions neurons against oxidative insult. J Neurosci 2004;24:1101-1112.

45. Shih AY, Johnson DA, Wong G, et al. Coordinate regulation of glutathione biosynthesis and release by Nrf2-expressing glia potently protects neurons from oxidative stress. J Neurosci 2003; 23:3394-3406.

46. Chen PC, Vargas MR, Pani AK, et al. Nrf2-mediated neuroprotection in the MPTP mouse model of Parkinson's disease: Critical role for the astrocyte. Proc Natl Acad Sci U S A 2009;106:29332938.

47. Clements CM, McNally RS, Conti BJ, Mak TW, Ting JP. DJ-1, a cancer- and Parkinson's disease-associated protein, stabilizes the antioxidant transcriptional master regulator Nrf2. Proc Natl Acad Sci U S A 2006;103:15091-15096.

48. Hague S, Rogaeva E, Hernandez D, et al. Early-onset Parkinson's disease caused by a compound heterozygous DJ-1 mutation. Ann Neurol 2003;54:271-274.

49. Bonifati V, Rizzu P, Van Baren MJ, et al. Mutations in the DJ-1 gene associated with autosomal recessive early-onset parkinsonism. Science 2003;299:256-259.

50. Bandopadhyay R, Kingsbury AE, Cookson MR, et al. The expression of DJ-1 (PARK7) in normal human CNS and idiopathic Parkinson's disease. Brain 2004;127:420-430.

51. Waak J, Weber SS, Waldenmaier A, et al. Regulation of astrocyte inflammatory responses by the Parkinson's disease-associated gene DJ-1. FASEB J 2009;23:2478-2489.

52. Grothe C, Timmer M. The physiological and pharmacological role of basic fibroblast growth factor in the dopaminergic nigrostriatal system. Brain Res Rev 2007;54:80-91.

53. Lin LF, Doherty DH, Lile JD, Bektesh S, Collins F. GDNF: a glial cell line-derived neurotrophic factor for midbrain dopaminergic neurons. Science 1993;260:1130-1132.

54. Saavedra A, Baltazar G, Santos P, Carvalho CM, Duarte EP Selective injury to dopaminergic neurons up-regulates GDNF in substantia nigra postnatal cell cultures: role of neuron-glia crosstalk. Neurobiol Dis 2006;23:533-542.

55. Petrova PS, Raibekas A, Pevsner J, et al. Discovering novel phenotype-selective neurotrophic factors to treat neurodegenerative diseases. Prog Brain Res 2004;146:168-183.

56. Deierborg T, Soulet D, Roybon L, Hall V, Brundin P. Emerging restorative treatments for Parkinson's disease. Prog Neurobiol 2008;85:407-432.

57. Nutt JG, Burchiel KJ, Comella CL, et al.; ICV GDNF Study Group. Randomized, double-blind trial of glial cell line-derived neurotrophic factor (GDNF) in PD. Neurology 2003;60:69-73.

58. Lang AE, Gill S, Patel NK, et al. Randomized controlled trial of intraputamenal glial cell line-derived neurotrophic factor infusion in Parkinson disease [Erratum in: Ann Neurol 2006;60:747]. Ann Neurol 2006;59:459-466.

59. Salvatore MF, Ai Y, Fischer B, et al. Point source concentration of GDNF may explain failure of phase II clinical trial. Exp Neurol 2006;202:497-505.

60. Voutilainen MH, Bäck S, Pörsti E, et al. Mesencephalic astrocytederived neurotrophic factor is neurorestorative in rat model of Parkinson's disease. J Neurosci 2009;29:9651-9659.

61. Abeliovich A, Schmitz Y, Farinas I, et al. Mice lacking $\alpha$-synuclein display functional deficits in the nigrostriatal dopamine system. Neuron 2000;25:239-252.

62. Polymeropoulos MH, Lavedan C, Leroy E, et al. Mutation in the $\alpha$-synuclein gene identified in families with Parkinson's disease. Science 1997;276:2045-2047.

63. Krüger R, Kuhn W, Müller T, et al. Ala30Pro mutation in the gene encoding $\alpha$-synuclein in Parkinson's disease. Nat Genet 1998; 18:107-108.

64. Zarranz JJ, Alegre J, Gómez-Esteban JC, et al. The new mutation, E46K, of $\alpha$-synuclein causes Parkinson and Lewy body dementia. Ann Neurol 2004;55:164-173.

65. Singleton AB, Farrer M, Johnson J, et al. $\alpha$-Synuclein locus triplication causes Parkinson's disease. Science 2003;302:841.

66. Weinreb PH, Zhen W, Poon AW, Conway KA, Lansbury PT Jr. NACP, a protein implicated in Alzheimer's disease and learning, is natively unfolded. Biochemistry 1996;35:13709-13715.

67. Savitt JM, Dawson VL, Dawson TM. Diagnosis and treatment of Parkinson disease: molecules to medicine. J Clin Invest 2006; 116:1744-1754.

68. Li JY, Englund E, Holton JL, et al. Lewy bodies in grafted neurons in subjects with Parkinson's disease suggest host-to-graft disease propagation. Nat Med 2008;14:501-503.

69. Kordower JH, Chu Y, Hauser RA, Freeman TB, Olanow CW Lewy body-like pathology in long-term embryonic nigral transplants in Parkinson's disease. Nat Med 2008;14:504-506.

70. Mendez I, Viñuela A, Astradsson A, et al. Dopamine neurons implanted into people with Parkinson's disease survive without pathology for 14 years. Nat Med 2008;14:507-509. 
71. Brundin P, Li JY, Holton JL, Lindvall O, Revesz T. Research in motion: the enigma of Parkinson's disease pathology spread. Nat Rev Neurosci 2008;9:741-745.

72. Desplats P, Lee HJ, Bae EJ, et al. Inclusion formation and neuronal cell death through neuron-to-neuron transmission of $\alpha$-synuclein [Erratum in: Proc Natl Acad Sci U S A 2009;106: 17606]. Proc Natl Acad Sci U S A 2009;106:13010-13015.

73. Lee HJ, Suk JE, Patrick C, et al. Direct transfer of $\alpha$-synuclein from neuron to astroglia causes inflammatory responses in synucleinopathies. J Biol Chem 2010;285:9262-9272.

74. Brundin P, Li JY, Holton JL, Lindvall O, Revesz T. Research in motion: the enigma of Parkinson's disease pathology spread. Nat Rev Neurosci 2008;9:741-745.

75. Olanow CW, Prusiner SB. Is Parkinson's disease a prion disorder? Proc Natl Acad Sci U S A 2009;106:12571-12572.

76. Chu Y, Kordower JH. Age-associated increases of $\alpha$-synuclein in monkeys and humans are associated with nigrostriatal dopamine depletion: Is this the target for Parkinson's disease? Neurobiol Dis 2007;25:134-149.

77. Koistinaho M, Lin S, Wu X, et al. Apolipoprotein E promotes astrocyte colocalization and degradation of deposited amyloidbeta peptides. Nat Med 2004;10:719-726.

78. Wyss-Coray T, Loike JD, Brionne TC, et al. Adult mouse astrocytes degrade amyloid-beta in vitro and in situ. Nat Med 2003; 9:453-457.

79. Langston JW, Irwin I. MPTP: current concepts and controversies. Clin Neuropharmacol 1986;9:485-507.

80. Heikkila RE, Sieber BA, Manzino L, Sonsalla PK. Some features of the nigrostriatal dopaminergic neurotoxin 1-methyl-4-phenyl1,2,3,6-tetrahydropyridine (MPTP) in the mouse. Mol Chem Neuropathol 1989;10:171-183.

81. Christine CW, Langston JW, Turner RS, Starr PA. The neurophysiology and effect of deep brain stimulation in a patient with 1-methyl-4-phenyl-1,2,3,6-tetrahydropyridine-induced parkinsonism. J Neurosurg 2009;110:234-238.

82. Cui M, Aras R, Christian WV, et al. The organic cation transporter-3 is a pivotal modulator of neurodegeneration in the nigrostriatal dopaminergic pathway. Proc Natl Acad Sci U S A 2009;106:8043-8048.

83. Javitch JA, D'Amato RJ, Strittmatter SM, Snyder SH. Parkinsonism-inducing neurotoxin, $N$-methyl-4-phenyl-1,2,3,6-tetrahydropyridine: uptake of the metabolite $N$-methyl-4-phenylpyridine by dopamine neurons explain selective toxicity. Proc Natl Acad Sci USA 1985;82:2173-2177.

84. Mayer RA, Kindt MV, Heikkila RE. Prevention of the nigrostriatal toxicity of 1-methyl-4-phenyl-1,2,3,6-tetrahydropyridine by inhibitors of 3,4-dihydroxyphenylethylamine transport. J Neurochem 1986;47:1073-1079.

85. Namura I, Douillet P, Sun CJ, Pert A, Cohen RM, Chiueh CC. $\mathrm{MPP}^{+}$(1-methyl-4-phenylpyridine) is a neurotoxin to dopamine-, norepinephrine- and serotonin-containing neurons. Eur J Pharmacol 1987;136:31-37.

86. McNaught KS, Carrupt PA, Altomare C, et al. Isoquinoline derivatives as endogenous neurotoxins in the aetiology of Parkinson's disease. Biochem Pharmacol 1998;56:921-933.

87. Mahy N, Andrés N, Andrade C, Saura J. Age-related changes of MAO-A and -B distribution in human and mouse brain. Neurobiology (Bp) 2000;8:47-54.

88. Fowler CJ, Wiberg A, Oreland L, Marcusson J, Winblad B. The effect of age on the activity and molecular properties of human brain monoamine oxidase. J Neural Transm 1980;49:1-20.

89. Mandel S, Grunblatt E, Riederer P, Youdim MB. Genes and oxidative stress in parkinsonism: cDNA microarray studies. Adv Neurol 2003;91:123-132.

90. Mallajosyula JK, Kaur D, Chinta SJ, et al. MAO-B elevation in mouse brain astrocytes results in Parkinson's pathology. PLoS One 2008;3:e1616.

91. Fowler JS, Volkow ND, Wang GJ, et al. Inhibition of monoamine oxidase B in the brains of smokers. Nature 1996;379:733-736.

92. Saccone NL, Rice JP, Rochberg N, et al. Genome screen for platelet monoamine oxidase (MAO) activity. Am J Med Genet 1999;88:517-521.
93. Parkinson Study Group. Effects of tocopherol and deprenyl on the progression of disability in early Parkinson's disease. N Engl J Med 1993;328:176-183.

94. Shoulson I, Oakes D, Fahn S, et al. Impact of sustained deprenyl (selegiline) in levodopa-treated Parkinson's disease: a randomized placebo-controlled extension of the deprenyl and tocopherol antioxidative therapy of parkinsonism trial. Ann Neurol 2002;51: 604-612.

95. Parkinson Study Group. Mortality in DATATOP: a multicenter trial in early Parkinson's disease. Ann Neurol 1998;43:318-325.

96. Olanow CW. Rationale for considering that propargylamines might be neuroprotective in Parkinson's disease. Neurology 2006;66 (Suppl 4):S69-S79.

97. Wakabayashi K, Hayashi S, Yoshimoto M, Kudo H, Takahashi H. NACP/ $\alpha$-synuclein-positive filamentous inclusions in astrocytes and oligodendrocytes of Parkinson's disease brains. Acta Neuropathol 2000;99:14-20.

98. Saijo K, Winner B, Carson CT, et al. A Nurr1/CoREST pathway in microglia and astrocytes protects dopaminergic neurons from inflammation-induced death. Cell 2009;137:47-59.

99. Kadkhodaei B, Ito T, Joodmardi E, et al. Nurr1 is required for maintenance of maturing and adult midbrain dopamine neurons. J Neurosci 2009;29:15923-15932.

100. Zetterstrom RH, Solomin L, Jansson L, Hoffer BJ, Olson L, Perlmann T. Dopamine neuron agenesis in Nurr1-deficient mice. Science 1997;276:248-250.

101. Jankovic J, Chen S, Le WD. The role of Nurr1 in the development of dopaminergic neurons and Parkinson's disease. Prog Neurobiol 2005;77:128-138.

102. Le WD, Xu P, Jankovic J, et al. Mutations in NR4A2 associated with familial Parkinson disease [Erratum in: Nat Genet 2003;33: 214]. Nat Genet 2003;33:85-89.

103. Grimes DA, Han F, Panisset M, et al. Translated mutation in the Nurrl gene as a cause for Parkinson's disease. Mov Disord 2006;21:906-909.

104. Jacobsen KX, MacDonald H, Lemonde S, et al. A Nurr1 point mutant, implicated in Parkinson's disease, uncouples ERK1/2dependent regulation of tyrosine hydroxylase transcription. Neurobiol Dis 2008;29:117-122.

105. Dubois C, Hengerer B, Mattes H. Identification of a potent agonist of the orphan nuclear receptor Nurr1. ChemMedChem 2006; 1:955-958.

106. Abbracchio MP, Burnstock G, Verkhratsky A, Zimmermann H. Purinergic signalling in the nervous system: an overview. Trends Neurosci 2009;32:19-29.

107. Boison D, Chen JF, Fredholm BB. Adenosine signaling and function in glial cells. Cell Death Differ 2010;17:1071-1082.

108. Rosin DL, Robeva A, Woodard RL, Guyenet PG, Linden J. Immunohistochemical localization of adenosine $\mathrm{A}_{2 \mathrm{~A}}$ receptors in the rat central nervous system. J Comp Neurol 1998;401:163186.

109. Schiffmann SN, Jacobs O, Vanderhaeghen JJ. Striatal restricted adenosine A2 receptor (RDC8) is expressed by enkephalin but not by substance $\mathrm{P}$ neurons: an in situ hybridization histochemistry study. J Neurochem 1991;57:1062-1067.

110. Schwarzschild MA, Agnati L, Fuxe K, Chen JF, Morelli M. Targeting adenosine $\mathrm{A}_{2 \mathrm{~A}}$ receptors in Parkinson's disease. Trends Neurosci 2006;29:647-654.

111. Verkhratsky A, Krishtal OA, Burnstock G. Purinoceptors on neuroglia. Mol Neurobiol 2009;39:190-208.

112. Pinna A, Fenu S, Morelli M. Motor stimulant effects of the adenosine $\mathrm{A}_{2 \mathrm{~A}}$ receptor antagonist $\mathrm{SCH} 58261$ do not develop tolerance after repeated treatments in 6-hydroxydopamine-lesioned rats. Synapse 2001;39:233-238.

113. Kanda T, Jackson MJ, Smith LA, et al. Adenosine $A_{2 A}$ antagonist: a novel antiparkinsonian agent that does not provoke dyskinesia in parkinsonian monkeys. Ann Neurol 1998;43:507-513.

114. Morelli M, Carta AR, Jenner P. Adenosine $A_{2 A}$ receptors and Parkinson's disease. Handb Exp Pharmacol 2009;193:589-615.

115. Pinna A. Novel investigational adenosine $A_{2 A}$ receptor antagonists for Parkinson's disease. Expert Opin Investig Drugs 2009; 18:1619-1631. 
116. Jenner P, Mori A, Hauser R, Morelli M, Fredholm BB, Chen JF. Adenosine, adenosine $\mathrm{A}_{2 \mathrm{~A}}$ antagonists, and Parkinson's disease. Parkinsonism Relat Disord 2009;15:406-413.

117. Ross GW, Abbott RD, Petrovitch H, et al. Association of coffee and caffeine intake with the risk of Parkinson disease. JAMA 2000;283:2674-2679.

118. Ascherio A, Zhang SM, Hernán MA, et al. Prospective study of caffeine consumption and risk of Parkinson's disease in men and women. Ann Neurol 2001;50:56-63.

119. Chen JF, Xu K, Petzer JP, et al. Neuroprotection by caffeine and $\mathrm{A}_{2 \mathrm{~A}}$ adenosine receptor inactivation in a model of Parkinson's disease. J Neurosci 2001;21:RC143.

120. Ikeda K, Kurokawa M, Aoyama S, Kuwana Y. Neuroprotection by adenosine $\mathrm{A}_{2 \mathrm{~A}}$ receptor blockade in experimental models of Parkinson's disease. J Neurochem 2002;80:262-270.

121. Pierri M, Vaudano E, Sager T, Englund U. KW-6002 protects from MPTP induced dopaminergic toxicity in the mouse. Neuropharmacology 2005;48:517-524.

122. Anderson CM, Bergher JP, Swanson RA. ATP-induced ATP release from astrocytes. J Neurochem 2004;88:246-256.

123. Bowser DN, Khakh BS. Vesicular ATP is the predominant cause of intercellular calcium waves in astrocytes. J Gen Physiol 2007; 129:485-491.

124. Dawson TM, Ko HS, Dawson VL. Genetic animal models of Parkinson's disease. Neuron 2010;66:646-661. 